\title{
An Identity Generator: Basic Commutators
}

\author{
M. Farrokhi D. G. \\ Institute of Mathematics \\ University of Tsukuba \\ Tsukuba Ibaraki 305, Japan \\ m.farrokhi.d.g@gmail.com
}

Submitted: Feb 23, 2008; Accepted: Apr 26, 2008; Published: May 5, 2008

Mathematics Subject Classification: Primary 05A19, 68R15; Secondary 11B39, 20E05

\begin{abstract}
We introduce a group theoretical tool on which one can derive a family of identities from sequences that are defined by a recursive relation. As an illustration it is shown that

$$
\sum_{i=1}^{n-1} F_{n-i} F_{i}^{2}=\frac{1}{2} \sum_{i=1}^{n}(-1)^{n-i}\left(F_{2 i}-F_{i}\right)=\left(\begin{array}{c}
F_{n+1} \\
2
\end{array}\right)-\left(\begin{array}{c}
F_{n} \\
2
\end{array}\right),
$$
\end{abstract}

where $\left\{F_{n}\right\}$ denotes the sequence of Fibonacci numbers.

\section{Preliminaries and Introduction}

We start our work with recalling some basic facts about the structural properties of words in a free group; cf. [1]. Let $F$ be the free group generated by the set $X=\left\{x_{1}, \ldots, x_{n}\right\}$. Marshall Hall [1] introduced a family of words in $F$, which are known as basic commutators and play an essential role. Every basic commutator $u$ has a weight, denoted by $\omega(u)$, which is a natural number. Also, the basic commutators can be ordered generally with respect to their weight.

Definition. (Basic Commutators)

1) $x_{1}, \ldots, x_{n}$ are basic commutators of weight 1 and are ordered with respect to each other (here $x_{1}<\cdots<x_{n}$ ),

2 ) if the basic commutators of weights less than $n$ are defined, then the basic commutators of weight $n$ are $w=[u, v]=u^{-1} v^{-1} u v$, where

i) $u, v$ are basic commutators and $\omega(u)+\omega(v)=n$,

ii) $u>v$ and if $u=[s, t]$ then $t \leq v$.

If $\omega(u)<n$ then $u<w$. The basic commutators of weight $n$ are ordered arbitrarily with respect to each other. 
The following theorem of Marshall Hall plays a basic role in the study of basic commutators. Recall that the commutator subgroups $\gamma_{k}(G)$ in a group $G$ are defined recursively by $\gamma_{1}(G)=G$ and

$$
\gamma_{i+1}(G)=\left[\gamma_{i}(G), G\right]=\left\langle[x, g] ; x \in \gamma_{i}(G), g \in G\right\rangle,
$$

for all $i \geq 1$. We refer the reader to [1] for some basic properties of $\gamma_{k}(G)$.

Theorem 1.1. (Marshall Hall [1, Theorem 11.2.4]) If $F$ is the free group with free generators $x_{1}, \ldots, x_{n}$ and if $c_{1}, \ldots, c_{m}$ is the sequence of basic commutators of weights $1, \ldots, k$, then an arbitrary element $w$ of $F$ has a unique representation

$$
w=c_{1}^{a_{1}} \cdots c_{m}^{a_{m}} \quad\left(\bmod \gamma_{k+1}(F)\right),
$$

where $a_{1}, \ldots, a_{m}$ are integers. Moreover, the basic commutators of weight $k$ form a basis for the free abelian group $\gamma_{k}(F) / \gamma_{k+1}(F)$.

In this paper, we introduce a general strategy on the discovery of almost number theoretical identities using a word-based combinatorics. As an illustration it is shown that

$$
\sum_{i=1}^{n-1} F_{n-i} F_{i}^{2}=\frac{1}{2} \sum_{i=1}^{n}(-1)^{n-i}\left(F_{2 i}-F_{i}\right)=\left(\begin{array}{c}
F_{n+1} \\
2
\end{array}\right)-\left(\begin{array}{c}
F_{n} \\
2
\end{array}\right),
$$

where $\left\{F_{n}\right\}$ denotes the sequence of Fibonacci numbers.

\section{Main Results}

To explain our method, let $F$ be the free group of finite rank generated by $X$ and $\left\{w_{n}\right\}$ be a recursively defined sequence of words in $F$. Also, let $k \geq 1$ and $c_{1}, \ldots, c_{m}$ be the sequence of basic commutators of weights $1, \ldots, k$. Then, by Theorem 1.1, $w_{n}$ has a unique representation

$$
w_{n}=c_{1}^{a_{1, n}} \cdots c_{m}^{a_{m, n}} \quad\left(\bmod \gamma_{k+1}(F)\right),
$$

where $a_{1, n}, \ldots, a_{m, n}$ are integers. Since $\left\{w_{n}\right\}$ is recursively defined, we may assume that $w_{n}=W_{n}\left(w_{1}, \ldots, w_{n-1}, X\right)$, where $W_{n}$ is a word on $w_{1}, \ldots, w_{n-1}$ and elements of $X$. Suppose that $i \geq 1$ and $a_{j, k}$ 's are known for all $j$ such that $\omega\left(c_{j}\right)<\omega\left(c_{i}\right)$ and all $k \geq 1$. Feeding the representation (1) of $w_{1}, \ldots, w_{n-1}$ in $w_{n}$ one observes that $a_{i, n}$ can be obtained recursively by $a_{i, 1}, \ldots, a_{i, n-1}$, i.e., $\left\{a_{i, n}\right\}_{n=1}^{\infty}$ is also a recursive sequence. Now, by solving the recursive sequences $\left\{w_{n}\right\}$ and $\left\{a_{i, n}\right\}_{n=1}^{\infty}$, we obtain $a_{i, n}$ in two different forms from which we obtain an identity. An identity which is obtained in this way is called the $c_{i}$ identity of $\left\{w_{n}\right\}$. It is evident that different methods in solving the sequences $\left\{w_{n}\right\}$ and $\left\{a_{i, n}\right\}_{n=1}^{\infty}$ would give different identities. To be more tangible what it means, in Theorem 2.2 we obtain a $[y, x]$-identity in details.

Throughout this paper, $F$ denotes the free group of rank 2 generated by $x$ and $y$. In this case, $x<y<[y, x]$ would denotes the basic commutators of weights $1,1,2$, 
respectively. In what follows we use frequently the well-known identities $y x=x y[y, x]$, $[x y, z]=[x, z]^{y}[x, z]$ and $[x, y z]=[x, z][x, y]^{z}$, where $x, y$ and $z$ are elements of an arbitrary group $G$. As a direct consequence of these identities we can prove

Lemma 2.1. For any group $G$ and elements $x, y \in G$

i) $y^{n} x^{m}=x^{m} y^{n}[y, x]^{m n} \quad\left(\bmod \gamma_{3}(G)\right)$;

ii) $(x y)^{n}=x^{n} y^{n}[y, x]^{\left(\begin{array}{c}n \\ 2\end{array}\right)} \quad\left(\bmod \gamma_{3}(G)\right)$.

Now, we explain the first example in details. Let $w_{1}=x^{a} y^{c}, w_{2}=x^{b} y^{d}$ and $w_{n+2}=$ $w_{n}^{u} w_{n+1}^{v}$, where $a, b, c, d, u, v$ are integers and $n \geq 0$. Also, let $\bar{F}=F / \gamma_{3}(F)$ and $\bar{w}=$ $w \gamma_{3}(F)$, for each $w \in F$. Then, by Theorem 1.1, there are unique integers $a_{n}, b_{n}$ and $c_{n}$ such that

$$
\bar{w}_{n}=\bar{x}^{a_{n}} \bar{y}^{b_{n}}[\bar{y}, \bar{x}]^{c_{n}},
$$

for all $n \geq 1$.

To obtain the $[y, x]$-identity of $\left\{w_{n}\right\}$ we need some more notations. To do this, let $\left\{L_{n}\right\},\left\{L_{n}^{\prime}\right\}$ be the sequences recursively defined by the rules $L_{n+2}=u L_{n}+v L_{n+1}$ and $L_{n+2}^{\prime}=u L_{n}^{\prime}+v L_{n+1}^{\prime}$, where $L_{0}=0, L_{1}=u, L_{0}^{\prime}=1, L_{1}^{\prime}=v$ and $n \geq 0$. Moreover, Let $\left\{G_{n}\right\},\left\{G_{n}^{\prime}\right\}$ be sequences recursively defined by $G_{n+2}=u G_{n}+v G_{n+1}$ and $G_{n+2}^{\prime}=$ $u G_{n}^{\prime}+v G_{n+1}^{\prime}$, where $G_{1}=a, G_{2}=b, G_{1}^{\prime}=c, G_{2}^{\prime}=d$ and $n \geq 1$.

Utilising the notations above, we have

Theorem 2.2.

$$
\begin{gathered}
\sum_{i=1}^{n} L_{n-i}^{\prime}\left[\left(\begin{array}{l}
u \\
2
\end{array}\right) G_{i} G_{i}^{\prime}+\left(\begin{array}{l}
v \\
2
\end{array}\right) G_{i+1} G_{i+1}^{\prime}+u v G_{i+1} G_{i}^{\prime}\right] \\
=u \sum_{i=1}^{n}(-u)^{n-i}\left[L_{i-1} L_{i-1}^{\prime}+v\left(\begin{array}{c}
L_{i-1}^{\prime} \\
2
\end{array}\right)\right]\left|\begin{array}{cc}
a & b \\
c & d
\end{array}\right|+a c\left(\begin{array}{c}
L_{n} \\
2
\end{array}\right)+b d\left(\begin{array}{c}
L_{n}^{\prime} \\
2
\end{array}\right)+b c L_{n} L_{n}^{\prime},
\end{gathered}
$$

for all $n \geq 1$

To prove Theorem 2.2, we need the following lemmas.

Lemma 2.3. If $n \geq 0$, then $L_{n+1}=u L_{n}^{\prime}$ and $L_{n+1}^{\prime}=L_{n}+v L_{n}^{\prime}$.

Proof. By definition $L_{1}=u=u L_{0}^{\prime}, L_{2}=u v=u L_{1}^{\prime}, L_{1}^{\prime}=v=L_{0}+v L_{0}^{\prime}$ and $L_{2}^{\prime}=u+v^{2}=$ $L_{1}+v L_{1}^{\prime}$. Now, if $n>1$ and the result hold for $n-2$ and $n-1$, then

$$
\begin{aligned}
& L_{n+2}=u L_{n}+v L_{n+1}=u\left(u L_{n-1}^{\prime}+v L_{n}^{\prime}\right)=u L_{n+1}^{\prime}, \\
& L_{n+2}^{\prime}=u L_{n}^{\prime}+v L_{n+1}^{\prime}=L_{n+1}+v L_{n+1}^{\prime},
\end{aligned}
$$

as required.

Lemma 2.4. Let $k$ and $n$ be nonnegative integers. Then

i) $\bar{w}_{n}^{k}=\bar{x}^{k a_{n}} \bar{y}^{k b_{n}}[\bar{y}, \bar{x}]^{k c_{n}+\left(\begin{array}{c}k \\ 2\end{array}\right) a_{n} b_{n}}$;

ii) $\left[\bar{w}_{n+1}, \bar{w}_{n}\right]=[\bar{y}, \bar{x}]^{(-u)^{n-1}(a d-b c)}$. 
Proof. i) It is obvious by Lemma 2.1(ii).

ii) If $n=1$, then $\left[\bar{w}_{n+1}, \bar{w}_{n}\right]=\left[\bar{w}_{2}, \bar{w}_{1}\right]=\left[\bar{x}^{b} \bar{y}^{d}, \bar{x}^{a} \bar{y}^{c}\right]=[\bar{y}, \bar{x}]^{a d-b c}$. Now, if $n>1$, then

$$
\left[\bar{w}_{n+1}, \bar{w}_{n}\right]=\left[\bar{w}_{n-1}^{u} \bar{w}_{n}^{v}, \bar{w}_{n}\right]=\left[\bar{w}_{n}, \bar{w}_{n-1}\right]^{-u}
$$

and the result follows inductively.

Proof of Theorem 2.2. To prove identity (2), we calculate $c_{n+2}$ in two different ways.

1) First, we count $c_{n+2}$ directly by solving $\left\{c_{n}\right\}$. If $n \geq 1$, then by Lemmas 2.1(i) and 2.4(i)

$$
\begin{aligned}
\bar{w}_{n+2} & =\bar{w}_{n}^{u} \bar{w}_{n+1}^{v} \\
& =\bar{x}^{u a_{n}} \bar{y}^{u b_{n}}[\bar{y}, \bar{x}]^{u c_{n}+\left(\begin{array}{c}
u \\
2
\end{array}\right) a_{n} b_{n}} \bar{x}^{v a_{n+1}} \bar{y}^{v b_{n+1}}[\bar{y}, \bar{x}]^{v c_{n+1}+\left(\begin{array}{c}
v \\
2
\end{array}\right) a_{n+1} b_{n+1}} \\
& =\bar{x}^{u a_{n}} \bar{y}^{u b_{n}} \bar{x}^{v a_{n+1}} \bar{y}^{v b_{n+1}}[\bar{y}, \bar{x}]^{u c_{n}+v c_{n+1}+\left(\begin{array}{c}
u \\
2
\end{array}\right) a_{n} b_{n}+\left(\begin{array}{c}
v \\
2
\end{array}\right) a_{n+1} b_{n+1}} \\
& =\bar{x}^{u a_{n}} \bar{x}^{v a_{n+1}} \bar{y}^{u b_{n}}[\bar{y}, \bar{x}]^{u v a_{n+1} b_{n}} \bar{y}^{v b_{n+1}}[\bar{y}, \bar{x}]^{u c_{n}+v c_{n+1}+\left(\begin{array}{c}
u \\
2
\end{array}\right) a_{n} b_{n}+\left(\begin{array}{c}
v \\
2
\end{array}\right) a_{n+1} b_{n+1}} \\
& =\bar{x}^{u a_{n}+v a_{n+1}} \bar{y}^{u b_{n}+v b_{n+1}}[\bar{y}, \bar{x}]^{u c_{n}+v c_{n+1}+\left(\begin{array}{c}
u \\
2
\end{array}\right) a_{n} b_{n}+\left(\begin{array}{c}
v \\
2
\end{array}\right) a_{n+1} b_{n+1}+u v a_{n+1} b_{n}} .
\end{aligned}
$$

Hence

$$
\begin{aligned}
a_{n+2} & =u a_{n}+v a_{n+1}, \\
b_{n+2} & =u b_{n}+v b_{n+1}, \\
c_{n+2} & =u c_{n}+v c_{n+1}+\left(\begin{array}{l}
u \\
2
\end{array}\right) a_{n} b_{n}+\left(\begin{array}{l}
v \\
2
\end{array}\right) a_{n+1} b_{n+1}+u v a_{n+1} b_{n} .
\end{aligned}
$$

It follows from the definitions of $\left\{a_{k}\right\},\left\{b_{k}\right\}$ and $\left\{G_{k}\right\},\left\{G_{k}^{\prime}\right\}$ that $a_{k}=G_{k}$ and $b_{k}=G_{k}^{\prime}$, for all $k \geq 1$. Let $d_{k+2}=\left(\begin{array}{c}u \\ 2\end{array}\right) a_{k} b_{k}+\left(\begin{array}{c}v \\ 2\end{array}\right) a_{k+1} b_{k+1}+u v a_{k+1} b_{k}$, for all $k \geq 1$. Then $c_{n+2}=$ $u c_{n}+v c_{n+1}+d_{n+2}=L_{1} c_{n}+L_{1}^{\prime} c_{n+1}+L_{0}^{\prime} d_{n+2}$. Now, suppose that $1 \leq k<n$ and

$$
c_{n+2}=L_{k} c_{n-k+1}+L_{k}^{\prime} c_{n-k+2}+L_{k-1}^{\prime} d_{n-k+3}+\cdots+L_{0}^{\prime} d_{n+2} \text {. }
$$

Then

$$
\begin{aligned}
c_{n+2} & =L_{k} c_{n-k+1}+L_{k}^{\prime} c_{n-k+2}+L_{k-1}^{\prime} d_{n-k+3}+\cdots+L_{0}^{\prime} d_{n+2} \\
& =L_{k} c_{n-k+1}+L_{k}^{\prime}\left(u c_{n-k}+v c_{n-k+1}+d_{n-k+2}\right)+L_{k-1}^{\prime} d_{n-k+3}+\cdots+L_{0}^{\prime} d_{n+2} \\
& =L_{k+1} c_{n-k}+L_{k+1}^{\prime} c_{n-k+1}+L_{k}^{\prime} d_{n-k+2}+\cdots+L_{0}^{\prime} d_{n+2}
\end{aligned}
$$

and so by induction we obtain

$$
\begin{aligned}
c_{n+2} & =L_{n} c_{1}+L_{n}^{\prime} c_{2}+L_{n-1}^{\prime} d_{3}+\cdots+L_{0}^{\prime} d_{n+2} \\
& =L_{n-1}^{\prime} d_{3}+\cdots+L_{0}^{\prime} d_{n+2}=\sum_{i=1}^{n} L_{n-i}^{\prime} d_{i+2},
\end{aligned}
$$

as $c_{1}=c_{2}=0$. Therefore

$$
c_{n+2}=\sum_{i=1}^{n} L_{n-i}^{\prime}\left[\left(\begin{array}{l}
u \\
2
\end{array}\right) G_{i} G_{i}^{\prime}+\left(\begin{array}{l}
v \\
2
\end{array}\right) G_{i+1} G_{i+1}^{\prime}+u v G_{i+1} G_{i}^{\prime}\right] .
$$


2) Now, we count $c_{n+2}$ in a different way by solving $\left\{w_{n}\right\}$. Put

$$
\alpha_{i}=(-u)^{n-i}\left[u L_{i-1} L_{i-1}^{\prime}+u v\left(\begin{array}{c}
L_{i-1}^{\prime} \\
2
\end{array}\right)\right]\left|\begin{array}{ll}
a & b \\
c & d
\end{array}\right|,
$$

for $i=1, \ldots, n$. Clearly $\alpha_{1}=0$ and so $\bar{w}_{n+2}=\bar{w}_{n}^{u} \bar{w}_{n+1}^{v}=\bar{w}_{n}^{L_{1}} \bar{w}_{n+1}^{L_{1}^{\prime}}[\bar{y}, \bar{x}]^{\alpha_{1}}$. We will show that for $i=1, \ldots, n$,

$$
\bar{w}_{n+2}=\bar{w}_{n-i+1}^{L_{i}} \bar{w}_{n-i+2}^{L_{i}^{\prime}}[\bar{y}, \bar{x}]^{\alpha_{1}+\cdots+\alpha_{i}} .
$$

If (4) holds for $i$, then using Lemmas 2.1(i,ii) and 2.4(ii)

$$
\begin{aligned}
& \bar{w}_{n+2}=\bar{w}_{n-i+1}^{L_{i}} \bar{w}_{n-i+2}^{L_{i}^{\prime}}[\bar{y}, \bar{x}]^{\alpha_{1}+\cdots+\alpha_{i}} \\
& =\bar{w}_{n-i+1}^{L_{i}}\left(\bar{w}_{n-i}^{u} \bar{w}_{n-i+1}^{v}\right)^{L_{i}^{\prime}}[\bar{y}, \bar{x}]^{\alpha_{1}+\cdots+\alpha_{i}}
\end{aligned}
$$

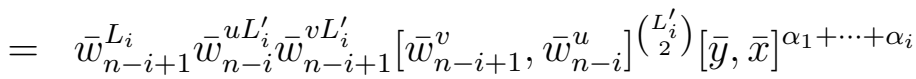

$$
\begin{aligned}
& =\bar{w}_{n-i+1}^{L_{i}} \bar{w}_{n-i}^{u L_{i}^{\prime}} \bar{w}_{n-i+1}^{v L_{i}^{\prime}}[\bar{y}, \bar{x}]^{\alpha_{1}+\cdots+\alpha_{i}+(-u)^{n-i-1} u v\left(\begin{array}{c}
L_{i}^{\prime} \\
2
\end{array}\right)(a d-b c)} \\
& =\bar{w}_{n-i}^{u L_{i}^{\prime}} \bar{w}_{n-i+1}^{L_{i}}\left[\bar{w}_{n-i+1}^{L_{i}}, \bar{w}_{n-i}^{u L_{i}^{\prime}}\right] \bar{w}_{n-i+1}^{v L_{i}^{\prime}}[\bar{y}, \bar{x}]^{\alpha_{1}+\cdots+\alpha_{i}+(-u)^{n-(i+1)} u v\left(\begin{array}{c}
L_{i}^{\prime} \\
2
\end{array}\right)(a d-b c)} \\
& =\bar{w}_{n-i}^{u L_{i}^{\prime}} \bar{w}_{n-i+1}^{L_{i}+v L_{i}^{\prime}}[\bar{y}, \bar{x}]^{\alpha_{1}+\cdots+\alpha_{i}+(-u)^{n-(i+1)}}\left(u L_{i} L_{i}^{\prime}+u v\left(\begin{array}{c}
L_{i}^{\prime} \\
2
\end{array}\right)\right)(a d-b c) \\
& =\bar{w}_{n-i}^{L_{i+1}} \bar{w}_{n-i+1}^{L_{i+1}^{\prime}}[\bar{y}, \bar{x}]^{\alpha_{1}+\cdots+\alpha_{i+1}} \text {. }
\end{aligned}
$$

By replacing $i$ by $n$ in (4) and using Lemma 2.1(i,ii), we get

$$
\begin{aligned}
\bar{w}_{n+2} & =\bar{w}_{1}^{L_{n}} \bar{w}_{2}^{L_{n}^{\prime}}[\bar{y}, \bar{x}]^{\alpha_{1}+\cdots+\alpha_{n}} \\
& =\left(x^{a} y^{c}\right)^{L_{n}}\left(x^{b} y^{d}\right)^{L_{n}^{\prime}}[\bar{y}, \bar{x}]^{\alpha_{1}+\cdots+\alpha_{n}} \\
& =x^{a L_{n}} y^{c L_{n}} x^{b L_{n}^{\prime}} y^{d L_{n}^{\prime}}[\bar{y}, \bar{x}]^{\alpha_{1}+\cdots+\alpha_{n}+a c\left(\begin{array}{c}
L_{n} \\
2
\end{array}\right)+b d\left(\begin{array}{c}
L_{n}^{\prime} \\
2
\end{array}\right)} \\
& =x^{a L_{n}+b L_{n}^{\prime}} y^{c L_{n}+d L_{n}^{\prime}}[\bar{y}, \bar{x}]^{\alpha_{1}+\cdots+\alpha_{n}+a c\left(\begin{array}{c}
L_{n} \\
2
\end{array}\right)+b d\left(\begin{array}{c}
L_{n}^{\prime} \\
2
\end{array}\right)+b c L_{n} L_{n}^{\prime}} .
\end{aligned}
$$

Therefore

$$
c_{n+2}=\alpha_{1}+\cdots+\alpha_{n}+a c\left(\begin{array}{c}
L_{n} \\
2
\end{array}\right)+b d\left(\begin{array}{c}
L_{n}^{\prime} \\
2
\end{array}\right)+b c L_{n} L_{n}^{\prime} .
$$

Now, the equations (3) and (5) imply the identity (2), which is the $[y, x]$-identity of $\left\{w_{n}\right\}$.

Corollary 2.5. For any $n>0$

$$
\sum_{i=1}^{n-1} F_{n-i} F_{i}^{2}=\frac{1}{2} \sum_{i=1}^{n}(-1)^{n-i}\left(F_{2 i}-F_{i}\right) .
$$

Proof. By putting $u=v=a=d=1$ and $b=c=0$ in identity (2), we get $L_{n}=F_{n}$, $L_{n}^{\prime}=F_{n+1}, G_{n}=F_{n-2}, G_{n}^{\prime}=F_{n-1}$ and so

$$
\sum_{i=1}^{n} F_{n+1-i} F_{i-1}^{2}=\sum_{i=1}^{n}(-1)^{n-i}\left(F_{i-1} F_{i}+\left(\begin{array}{c}
F_{i} \\
2
\end{array}\right)\right) \text {. }
$$


Now, $\sum_{i=1}^{n} F_{n+1-i} F_{i-1}^{2}=\sum_{i=1}^{n-1} F_{n-i} F_{i}^{2}$ and $F_{i-1} F_{i}+\left(\begin{array}{c}F_{i} \\ 2\end{array}\right)=\frac{1}{2}\left(F_{2 i}-F_{i}\right)$, which completes the proof.

Corollary 2.6. For any $n>0$

$$
\sum_{i=1}^{n-1} F_{n-i} F_{i} F_{i+1}=\left(\begin{array}{c}
F_{n+1} \\
2
\end{array}\right) .
$$

Proof. Put $u=v=a=b=d=1$ and $c=0$ in identity (2).

Corollary 2.7. For any $n>0$

$$
\sum_{i=1}^{n-1} F_{n-i} F_{i}^{2}=\left(\begin{array}{c}
F_{n+1} \\
2
\end{array}\right)-\left(\begin{array}{c}
F_{n} \\
2
\end{array}\right)
$$

Proof. By Corollary 2.6, we have

$$
\begin{aligned}
\sum_{i=1}^{n-1} F_{n-i} F_{i}^{2} & =\sum_{i=1}^{n-1} F_{n-i} F_{i}\left(F_{i+1}-F_{i-1}\right) \\
& =\sum_{i=1}^{n-1} F_{n-i} F_{i} F_{i+1}-\sum_{i=1}^{n-1} F_{n-i} F_{i} F_{i-1} \\
& =\sum_{i=1}^{n-1} F_{n-i} F_{i} F_{i+1}-\sum_{i=1}^{n-2} F_{n-1-i} F_{i} F_{i+1} \\
& =\left(\begin{array}{c}
F_{n+1} \\
2
\end{array}\right)-\left(\begin{array}{c}
F_{n} \\
2
\end{array}\right) .
\end{aligned}
$$

Similar to Corollary 2.7, one we can prove the following result.

Corollary 2.8. For any $n>0$

$$
\sum_{i=1}^{n-1} F_{n-i} F_{2 i}=\left(\begin{array}{c}
F_{n} \\
2
\end{array}\right)+\left(\begin{array}{c}
F_{n+1} \\
2
\end{array}\right) .
$$

Acknowledgment. The author would like to thank the referee for some useful suggestions and corrections.

\section{References}

[1] M. Hall, The Theory of Groups, Macmillan, New York, 1955. 